\title{
Retraite, activités non professionnelles et vieillissement cognitif Une exploration à partir des données de Share
}

\author{
Stéphane Adam*, Éric Bonsang**, Sophie Germain* \\ et Sergio Perelman**
}

Une approche en termes de frontières d'efficacité peut être utilisée pour étudier les relations entre les fonctions cognitives des personnes âgées de plus de 50 ans en Europe d'une part, et différents facteurs, plus particulièrement l'âge, l'éducation et l'exercice d'activités, professionnelles ou non, d'autre part. Le but est de construire une « frontière » correspondant au fonctionnement cognitif optimal que chacun des individus est censé atteindre étant donné son âge et son niveau d'éducation. À cette fin, nous utilisons des données individuelles collectées durant la première vague de l'enquête internationale et interdisciplinaire Share de 2004, laquelle contient le résultat de tests cognitifs réalisés auprès de plus de 22000 individus âgés, ainsi que des informations sur leur état de santé, mentale et physique, leur situation socio-économique, leur entourage familial, l'exercice d'activités professionnelles ou non professionnelles, l'isolement social et les performances cognitives individuelles mesurées à l'aide de tests. En plus du rôle fondamental joué par l'éducation face au vieillissement cognitif, le fait de rester en activité, ainsi que la pratique d'une activité non professionnelle ou d'activités physiques, vigoureuses ou modérées, sont positivement associés à la constitution des « réserves cognitives » individuelles. La mise à disposition des données des vagues successives de Share, prévues tous les deux ans auprès des mêmes individus, pourrait permettre de déterminer les liens de causalité subjacents.

* Service de Neuropsychologie, Université de Liège.

${ }^{*}$ CREPP, HEC-Ecole de Gestion, Université de Liège.

Les auteurs tiennent à remercier la Communauté Française de Belgique (Action de Recherche Concertée, ARC 05/10-332) pour son soutien financier. 
$\mathbf{L}$ a crise du milieu des années 1970 et le chômage de masse qui a suivi ont conduit de nombreux pays européens à favoriser la retraite anticipée en tant que moyen d'absorber l'excès d'offre sur le marché du travail. L'attrait des régimes de préretraite est attesté par les faibles taux d'emploi observés jusqu'à ce jour parmi la population âgée entre 55 et 64 ans dans de nombreux pays. La preuve que ce retrait de la vie active des travailleurs âgés aurait servi à augmenter le taux d'emploi des jeunes générations n'a cependant pas été faite. Au contraire, comme le montrent Blöndal et Scarpetta (1998), les pays qui n'ont pas suivi cette tendance, en Scandinavie notamment, ont réussi à maintenir des taux d'emploi élevés aussi bien parmi les travailleurs âgés que parmi toutes les autres catégories de la population, y compris les plus jeunes.

Il ne s'agit pas ici d'entrer dans ce débat mais d'étudier un aspect particulier de la vie des personnes âgées en rapport direct avec la fin de la vie active et leur bien-être : l'évolution des capacités cognitives face au vieillissement.

Il est actuellement largement reconnu que l'avancée en âge entraîne des changements structurels dans le cerveau qui, à leur tour, vont avoir un impact sur le fonctionnement cognitif de l'individu (1). Toutefois, ces modifications ne sont pas similaires chez tout le monde. Cette hétérogénéité est partiellement expliquée par des facteurs tels que le niveau d'éducation ou la pratique d'activités, professionnelles ou non (Stern, 2002).

Les informations collectées dans le cadre de la première vague de l'enquête internationale et interdisciplinaire Share (Survey on Health, Ageing and Retirement in Europe) permettent une mise en perspective des capacités cognitives des répondants (mesurées à partir de tests de mémoire, de fluence verbale, d'orientation et de capacités de calcul) et des informations détaillées sur leur situation socio-économique et occupationnelle, ainsi que sur leur état de santé et leur entourage familial, parmi d'autres.

Basée sur un vaste échantillon représentatif de la population âgée de 50 ans et plus répartie dans une dizaine de pays européens en 2004 (Börsch-Supan et al., 2005), notre exploitation de cette enquête par la méthode des frontières d'efficacité va témoigner du rôle important de l'âge, de l'éducation et de l'exercice d'activités sur les capacités cognitives.

\section{Le concept de réserve cognitive pour expliquer la non-uniformité du déclin cognitif}

$\mathrm{Au}$ cours des dernières décennies, un grand nombre de preuves se sont accumulées indiquant que le vieillissement s'accompagne d'un déclin de la performance dans un grand nombre de tâches cognitives, aussi bien dans des conditions de laboratoire que dans la vie de tous les jours (2). Par exemple, il est actuellement largement admis que l'âge influence quelques facteurs généraux tels que la vitesse de traitement de l'information, l'inhibition (c'est-à-dire la capacité à résister à l'information interférente) et la mémoire de travail (également appelée mémoire à court terme). Ceux-ci, à leur tour, vont influencer d'autres fonctions cognitives, comme la mémoire épisodique et le langage.

Ce déclin des fonctions cognitives avec l'âge est associé à des changements dans le cerveau. Cependant, ce déclin n'est pas uniforme, la nature fournissant dans ce sens des exemples de personnes âgées qui maintiennent leur vitalité cognitive, même à des âges très avancés.

En se basant sur ces observations, Stern (2002) et Scarmeas et Stern (2003) proposent le concept de « réserve cognitive » pour rendre compte de l'effet différentiel de l'âge ou de pathologies comme la maladie d'Alzheimer sur le fonctionnement cognitif. Ainsi, ce concept reflète le fait que l'intelligence innée, ou certains aspects liés aux expériences de la vie tels que le niveau d'éducation et les activités occupationnelles, produisent une forme de « réserve » prenant la forme d'un ensemble d'habiletés ou de répertoires qui permet à certaines personnes de limiter les répercussions du déclin cognitif associé soit à l'âge soit à la maladie d'Alzheimer.

Cependant, les processus conduisant à la formation de cette réserve demeurent encore relativement peu connus. Deux hypothèses, « passive » et " active », ont été avancées afin d'expliquer les substrats neurophysiologiques de la réserve cognitive. Selon l'hypothèse passive, une réserve cognitive importante serait le reflet d'une densité de synapses accrue et d'un

1. La cognition regroupe les divers processus mentaux allant de l'analyse perceptive à la commande motrice (en passant par la mémorisation, le raisonnement, l'attention, le langage...). Elle regroupe donc les fonctions de l'esprit humain par lesquelles nous construisons une représentation opératoire de la réalité à partir de nos perceptions, susceptible en particulier de nourrir nos raisonnements et guider nos actions.

2. Pour une revue récente de la littérature: Dixon et al. (2004) et Adam et al. (2006). 
nombre plus important de neurones, laissant une plus grande quantité de neurones disponibles lorsque certains sont altérés par un processus pathologique. L'hypothèse active quant à elle stipule que la réserve cognitive prendrait la forme d'une utilisation plus efficace ou plus flexible des réseaux cérébraux ou des paradigmes cognitifs, les rendant moins susceptibles à la détérioration (Stern, 2003).

Au-delà de ce débat, des études récentes ont tenté d'identifier des facteurs qui pourraient contribuer au développement de la réserve cognitive. En premier lieu, c'est le niveau d'éducation qui est largement reconnu comme ayant un impact significatif sur le fonctionnement cognitif (Le Carret et al., 2003). Mais d'autres facteurs participent aussi à la constitution de la réserve cognitive chez la personne âgée comme le type d'activités professionnelles ou de loisirs (Wilson et al., 2002), la complexité intellectuelle du travail (Schooler et al., 1999) ou un style de vie «actif» (Newson et Kemps, 2005).

\section{Une méthode d'estimation fondée sur les « frontières d'efficacité »}

C'est en prenant comme point de départ l'ensemble de ces recherches que nous proposons ici un test statistique qui fait appel à la méthodologie des frontières d'efficacité et aux données disponibles dans Share. L'objet de ce test est de permettre l'identification et la mesure de la réserve cognitive, potentielle et réalisée, et l'effet des facteurs qui agissent sur elle. Ce concept peut être illustré de manière très schématique dans un contexte de production simple, avec un input et un output (cf. graphique I).

Nous utilisons ici une extension de la méthode SFA (Stochastic Frontier Analysis), proposée par Battesse et Coelli (1995) qui permet de tester simultanément l'effet des variables explicatives sur les distances individuelles par rapport à la frontière d'efficacité (cf. encadré 1).

L'estimation de la frontière repose sur une fonction dépendant essentiellement de l'âge et des années d'éducation. Les facteurs explicatifs de la distance à la frontière incluent quant à eux une série de variables portant essentiellement sur le style de vie, en particulier l'exercice d'activités, au sens large, et sur le temps écoulé depuis le départ à la retraite pour les personnes ayant quitté définitivement leurs activités professionnelles (3).

\section{Une évaluation possible des fonctions exécutives et de la mémoire épisodique par des tests cognitifs}

Share est une enquête interdisciplinaire portant sur plus de 22000 individus âgés de 50 ans et plus interviewés en 2004 dans une dizaine de pays européens (4). Cette enquête, dont la deuxième vague était en cours de réalisation en 2006-2007, rassemble des données propres à différentes disciplines incluant la démographie, l'économie, l'épidémiologie, la psychologie et la sociologie.

Les fonctions cognitives sont mesurées en utilisant des tests simples d'orientation, de mémoire, de fluence verbale et de calcul (cf. encadré 2). Les répondants doivent également évaluer subjectivement leurs capacités de lecture et d'écriture. Cependant, dans la suite de cette étude,

\footnotetext{
3. Pays présents dans la version 1 de la première vague d'enquête de Share en 2004: Allemagne, Autriche, Danemark, Espagne, France, Grèce, Italie, Pays-Bas, Suède et Suisse. Deux autres pays, la Belgique et Israël, ont également réalisé cette première vague d'enquête mais en 2005 , raison pour laquelle ils ne font pas partie de la version 1.

4. La même méthodologie a été appliquée aux données de l'enquête Share dans Adam et al. (2006). Les différences entre cet article et l'article présent portent sur le choix de certaines variables explicatives, notamment en rapport avec le départ à la retraite et les maladies et accidents cérébraux, et sur la définition de l'échantillon lui-même, lequel correspond ici, à quelques exceptions près, à l'ensemble des personnes interrogées.
}

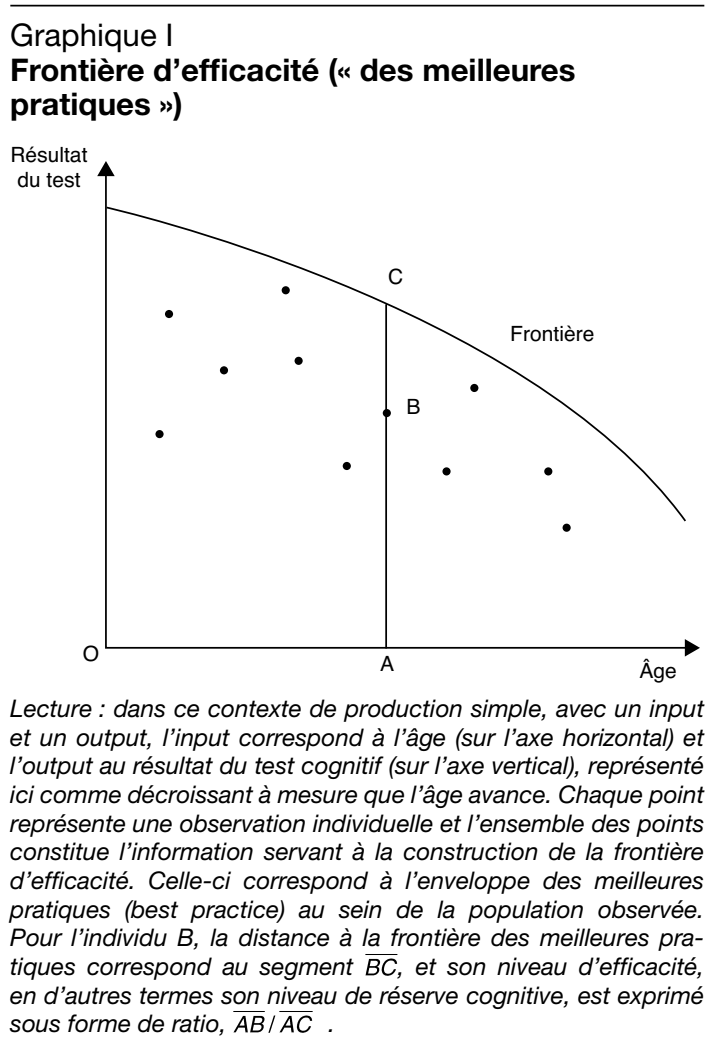


Encadré 1

\section{FRONTIÈRES D'EFFICACITÉ}

Différentes méthodes sont proposées dans la littérature pour la construction de frontières d'efficacité à partir des informations disponibles: les méthodes non paramétriques faisant appel à des techniques de programmation linéaire et les méthodes paramétriques faisant appel aux techniques économétriques. Parmi les méthodes non paramétriques, la plus utilisée est la méthode DEA (Data Envelopment Analysis), déterministe par construction, introduite par Charnes et al. (1978). Concernant les méthodes paramétriques, la méthode SFA (Stochastic Frontier Analysis), qui comme son nom l'indique est une méthode stochastique, est celle qui est privilégiée. Elle a été introduite simultanément par Aigner et al. (1977) et Meeusen et van den Broeck (1977). Nous utilisons ici une extension de la méthode SFA, proposée par Battese et Coelli (1995) qui permet de tester simultanément l'effet des variables explicatives sur les distances individuelles par rapport à la frontière d'efficacité. Une présentation détaillée de cette méthode et des différentes approches proposées depuis sont disponibles dans Kumbhakar et Lovell (2000) et Coelli et al. (2005).

Afin d'identifier les facteurs qui agissent sur les capacités cognitives, on propose une frontière paramétrique stochastique de la forme suivante :

$\ln r_{i}=f\left(X_{i}, D_{i}\right)+\varepsilon_{i}$

où In $r_{i}$ est le logarithme du score obtenu par l'individu $i$ au test cognitif ; $X_{i}$ un vecteur où l'on retrouve les deux facteurs identifiés comme déterminant les capacités cognitives dans la littérature en neuropsychologie: l'âge et l'éducation ; $D_{i}$ un vecteur contenant des variables de contrôle, et $\varepsilon_{i}$ un terme d'erreur composé de la forme :

$\varepsilon_{i}=v_{i}-u_{i}$

où $v_{i}$ est un terme aléatoire ayant une distribution symétrique supposée correspondre au bruit stochastique normal i.i.d. $N\left(0, \sigma_{v}^{2}\right)$ et $u_{i}$ un terme aléatoire supposé être distribué de manière indépendante et tronqué à zéro $N\left(\varphi_{i}, \sigma_{u}^{2}\right)$. Les deux termes sont supposés être distribués de manière indépendante $\left(\sigma_{u v}=0\right)$.

Dans la littérature consacrée aux frontières d'efficacité, le terme $u_{i}$ a une interprétation immédiate, il correspond à la distance par rapport à la meilleure pratique, représentée par la frontière stochastique $\left[f\left(X_{i}, D_{i}\right)+v_{i}\right]$. Dans le cas qui nous occupe, la meilleure pratique correspondra au score maximum, en termes de réserve cognitive, que chaque individu est supposé pouvoir atteindre étant donnés son âge et son niveau d'éducation, et le terme $u_{i}$ une mesure de la distance (efficacité) entre le score obtenu et l'optimum à la frontière.

Nous choisissons la forme trans-logarithmique (fonction translog) pour la spécification de la relation entre les fonctions cognitives d'une part, et l'âge $\left(x_{1}\right)$ et l'éducation $\left(x_{2}\right)$ en années d'étude d'autre part. La fonction translog correspond à une approximation de deuxième ordre de la fonction subjacente basée sur ces deux variables. À côté d'elles, on trouve des variables de contrôle $d_{m, i}(m=1,2, \ldots, M)$. La fonction à estimer a la forme :

$$
\begin{aligned}
\ln r_{i}= & \beta_{0}+\beta_{1} \ln x_{1, i}+\beta_{2} \ln x_{2, i}+0.5 \beta_{3}\left(\ln x_{1, i}\right)^{2}+0.5 \beta_{4}\left(\ln x_{2, i}\right)^{2}+\beta_{5}\left(\ln x_{1, i}\right)\left(\ln x_{2, i}\right) \\
& +\sum_{m=1}^{M} \lambda_{m} d_{m, i}+v_{i}-u_{i}
\end{aligned}
$$

où $\beta_{k}(k=0,1, \ldots, 5)$ et $\lambda_{m}(m=1,2, \ldots, M)$ sont des paramètres à estimer.

On remarquera que les termes $v_{i}$ et $u_{i}$ sont additionnés dans l'équation (3), comme dans l'équation (1), de manière indépendante à la transformation logarithmique. Dès lors, exp(-u) correspond au ratio d'efficacité $\left.0 \leq \exp \left(-u_{i}\right) \leq 1\right)$, illustré par le ratio $\overline{A B} / \overline{A C}$ du graphique I.

Un des avantages de la spécification de la forme translog est sa flexibilité. En plus de la transformation logarithmique des variables, les termes de second ordre autorisent des relations non linéaires et des interactions entre l'âge et les années d'éducation. De ce fait, la dérivée de la fonction translog à chaque point correspond au taux de substitution entre l'âge et l'éducation. En d'autres termes, on pourra répondre à la question suivante : combien chaque année d'éducation supplémentaire permet de compenser d'années de vieillissement cognitif ?

Nous faisons appel ici à une extension du modèle SFA, proposée par Battese et Coelli (1995), qui permet de tester simultanément l'effet d'autres variables explicatives, $z_{j, i}(j=1,2 \ldots, J)$, mais directement sur les distances à la frontière. Autrement dit sur l'efficacité cognitive individuelle, indiquée par $u_{i}$, au travers du paramètre de troncature $\phi_{i}$, comme suit :

$\varphi_{i}=\delta_{0}+\sum_{j=1}^{J} \delta_{j} z_{j, i}$. 


\section{Encadré 1 (suite)}

Les paramètres $\delta_{0}$ et $\delta_{j}$ dans l'équation (4) sont estimés conjointement avec les $\beta_{k}$ et $\lambda_{m}$ dans l'équation (3) en faisant appel à un algorithme d'optimisation du maximum de vraisemblance. Pour procéder à ces estimations nous avons utilisé le programme Frontier version 4.1, développé par Coelli (1994).

Enfin, il faut aussi noter que deux autres paramètres sont estimés simultanément : la variance du terme d'erreur composée : $\sigma_{\varepsilon}^{2}=\sigma_{v}^{2}+\sigma_{u}^{2}$ et la part de la variance du terme d'inefficience dans la variance totale : $\gamma=\sigma_{u}^{2} / \sigma_{\varepsilon}^{2}$. Par ailleurs, étant donné la nature stochastique de la frontière d'efficacité, les mesures d'efficacité obtenues prennent la forme d'espérances conditionnelles $E\left[\exp \left(-u_{i}\right) \mid \varepsilon_{i}\right]$.

Encadré 2

\section{QUESTIONS COGNITIVES DANS SHARE}

Les données ont été collectées en face-à-face par des enquêteurs munis d'ordinateurs portables faisant dérouler un questionnaire Capi, pour "Computer Assisted Personal Interviewing ", écrit dans le langage de programmation Blaise (Börsch-Supan et al. (2005) et Börsch-Supan et Jürges (2005), pour plus de détails sur la procédure d'échantillonnage, le questionnaire ou la méthodologie utilisés).

Nous reproduisons ici les questions, y compris les instructions aux enquêteurs, se rapportant aux deux tests cognitifs utilisés dans cette étude, telles qu'elles ont été posées aux répondants. Les questions CF007 et CF008 correspondent au test d'apprentissage et mémorisation (mémoire épisodique) et les questions CF009 et CF010 au test de fluence verbale (fonctions exécutives). Le deuxième essai du test d'apprentissage et mémorisation (non reproduit ici) est réalisé après une période de cinq minutes, approximativement.

CF007 : Apprentissage et mémorisation d'une liste de dix mots - Introduction

"Je vais maintenant vous lire une liste de mots qui vont s'afficher à l'écran de mon ordinateur. Nous avons volontairement choisi une liste assez longue, de sorte qu'il soit difficile de se souvenir de tous les mots. La plupart des gens ne s'en rappellent que quelques-uns. Merci d'écouter attentivement, car la liste de mots ne pourra être répétée. Quand j'aurai terminé, je vous demanderai de me dire à voix haute tous les mots dont vous vous souviendrez, dans n'importe quel ordre. Avez-vous bien compris ce que vous avez à faire? ”

CF008: Apprentissage et mémorisation d'une liste de dix mots - Premier essai

"Indiquez-moi à présent tous les mots dont vous vous souvenez. »

Instructions pour l'enquêteur : attendre jusqu'à ce que tous les mots se soient affichés à l'écran. Écrire les mots sur la feuille fournie. Laisser à la personne interrogée jusqu'à une minute pour se rappeler. Entrer les mots dont la personne se souvient correctement.

1. Beurre

2. Bras

3. Lettre

4. Reine

5. Ticket

6. Herbe

7. Coin

8. Pierre

9. Livre

10. Bâton

96. Aucun de ces mots

\section{CF009 Fluence verbale - Introduction}

" J'aimerais à présent que vous me donniez autant de noms d'animaux qu'il vous en vient à l'esprit. Vous disposez très exactement d'une minute. Prêt ? Allez-y! "

Instructions pour l'enquêteur: accorder très précisément une minute. Si le sujet s'arrête avant la fin du délai imparti, l'encourager à trouver d'autres noms. S'il reste silencieux pendant quinze secondes, répéter l'instruction de base (" Je veux que vous me donniez autant de noms d'animaux que possible »). Ne pas accorder de délai supplémentaire même si l'instruction doit être répétée.

\section{CF010 Fluence verbale - Score}

Instructions pour l'enquêteur: le score correspond à la somme de tous les noms d'animaux acceptables. Tout membre du règne animal, qu'il soit réel ou mythique, est considéré comme correct, exception faite des répétitions et des noms propres. Sont considérés comme corrects : les noms d'espèce et différents types au sein de cette espèce; nom du mâle, de la femelle et de la progéniture au sein d'une espèce. nous avons décidé de nous baser uniquement sur des tests correspondant à deux fonctions cognitives : les fonctions exécutives et la mémoire épisodique.
Les fonctions exécutives renvoient à un ensemble assez hétérogène de processus cognitifs de haut niveau dont le rôle principal est de faciliter l'adaptation du sujet aux situations non routi- 
nières, conflictuelles ou complexes (c'est-à-dire des situations où nos «automatismes » et nos habilités sur-apprises ne sont plus suffisants). En effet, si la plupart de nos activités quotidiennes peuvent être réalisées de façon routinière et sans y prêter attention (comme par exemple faire le trajet en voiture entre son domicile et son lieu de travail), un certain nombre de situations (en particulier des situations nouvelles comme par exemple rouler pour la première fois en voiture dans Paris) nécessitent par contre, pour produire un comportement adapté, l'intervention de mécanismes de contrôle (ou fonctions exécutives). Ces fonctions regroupent un grand nombre de processus distincts tels que : la capacité à résister à l'interférence, la planification de l'action, la génération d'hypothèses, la flexibilité cognitive, la prise de décisions, etc. La mémoire épisodique, partie de la mémoire à long terme, nous permet de stocker et de récupérer les événements que nous avons personnellement vécus (que ce soit le stockage d'une liste de mots au cours d'une expérience de psychologie ou ce que nous avons mangé au restaurant deux jours auparavant) ou des informations que nous avons apprises dans un contexte spatial et temporel bien précis (par exemple : nos dernières vacances, le dernier film vu au cinéma ou se souvenir de ce que notre voisin nous a dit lors de la soirée au restaurant deux jours auparavant). Il s'agit du système de mémoire auquel nous faisons généralement allusion lorsque nous parlons de « mémoire ».

Ce choix repose sur deux arguments. D'un point de vue psychométrique, nous avons sélectionné des tests sensibles (c'est-à-dire non sujets à des problèmes d'effets plafond ou plancher). D'un point de vue théorique, il est largement reconnu que les fonctions exécutives et la mémoire épisodique sont deux domaines cognitifs particulièrement affectés par le vieillissement. En fait, certains auteurs considèrent que ces deux aspects sont les premières fonctions cognitives qui déclinent avec 1'âge (Souchay et al., 2000 ; Anderson et Craik, 2000 ; Prull et al., 2000).

Les fonctions exécutives sont évaluées à l'aide d'un test de fluence verbale qui consiste à évaluer la rapidité du participant à citer des mots provenant d'une catégorie particulière. Lors de la première vague de Share, la personne interrogée est invitée à mentionner pendant une minute le plus grand nombre de noms d'animaux, le temps étant contrôlé par l'enquêteur à l'aide de son ordinateur. La performance est définie comme étant le nombre total de noms d'animaux différents donnés par le participant.
Les répétitions et les redondances (par exemple, « vache blanche », " vache brune ») n'étaient pas comptabilisées, ainsi que les noms propres (« Bambi », « Mickey »). Cependant, différents types de race (« caniche », « labrador») ou des noms spécifiques au genre ou à la génération (par exemple, « taureau », « vache », « veau ») étaient acceptés.

Le test concernant la mémoire épisodique est un test d'apprentissage et de rappel verbal pour lequel les participants doivent apprendre une liste de dix mots communs (cf. encadré 2). À un moment donné de l'enquête, les mots sont présentés automatiquement sur l'écran de l'ordinateur et l'enquêteur doit lire chacun de ces mots à haute voix. La personne interviewée est invitée ensuite à rappeler ces mots à deux moments différents : une première fois immédiatement après lecture de la liste et une deuxième fois après une courte période de plus ou moins cinq minutes pendant laquelle d'autres tests (des fonctions exécutives et de calcul) lui sont proposés. Les répondants peuvent citer les mots dans le désordre. Leur score est obtenu en additionnant le nombre de mots corrects récités durant la première et la deuxième phase du test (le résultat se situant entre 0 et 20 ).

Sur la base des scores obtenus à ces deux tests, nous avons composé un indice global correspondant, pour chaque individu, à la moyenne des scores standardisés des fonctions exécutives et de mémoire épisodique. De cette manière, nous obtenons une valeur représentant une évaluation plus globale et plus sensible du fonctionnement cognitif qui sera également utilisée dans la suite de cette étude.

On observe que les scores moyens des tests de fonctions exécutives, de mémoire épisodique et l'indice global diminuent avec l'âge et augmentent avec les années d'études (cf. tableau 1).

Ces tendances ont par ailleurs bien été vérifiées au niveau de chaque pays et par genre. Bien que celles-ci ne reposent que sur des données en coupe instantanée, elles illustrent de manière que nous estimons assez convaincante le rôle de l'âge et du niveau d'éducation sur le maintien des capacités cognitives face au vieillissement.

\section{Une multiplicité des variables mobilisables}

En accord avec le modèle à estimer, nous devons faire une distinction entre trois catégories de variables explicatives (cf. encadré 1). D'une part, au sein de la frontière stochastique, on 
Tableau 1

Performances cognitives moyennes

\begin{tabular}{|l|r|c|c|c|}
\hline Catégories & $N$ & $\begin{array}{c}\text { Fonctions } \\
\text { exécutives }\end{array}$ & $\begin{array}{c}\text { Mémoire } \\
\text { épisodique }\end{array}$ & $\begin{array}{r}\text { Indice } \\
\text { global }\end{array}$ \\
\hline \multicolumn{5}{|l}{ Classes d'âge } \\
\hline $50-54$ & 3697 & 20,9 & 9,8 & 0,34 \\
$55-59$ & 3836 & 20,6 & 9,4 & 0,27 \\
$60-64$ & 3684 & 19,8 & 8,8 & 0,16 \\
$65-69$ & 3244 & 18,8 & 8,2 & $-0,01$ \\
$70-74$ & 2558 & 17,1 & 7,3 & $-0,26$ \\
$75-79$ & 1910 & 16,1 & 6,7 & $-0,43$ \\
$80-84$ & 1105 & 15,0 & 5,9 & $-0,66$ \\
$85-89$ & 431 & 13,6 & 5,0 & $-0,95$ \\
$90-94$ & 167 & 12,5 & 4,3 & $-1,23$ \\
$95-99$ & 26 & 14,0 & 4,3 & $-1,10$ \\
\hline Années d'éducation & \multicolumn{4}{|l|}{} \\
\hline $0-2$ & 1248 & 12,3 & 5,0 & $-1,03$ \\
$3-5$ & 2684 & 14,3 & 6,1 & $-0,64$ \\
$6-9$ & 4702 & 17,5 & 7,6 & $-0,17$ \\
$10-12$ & 4897 & 20,1 & 9,0 & 0,20 \\
$13-15$ & 4807 & 21,7 & 9,6 & 0,37 \\
16 et plus & 2320 & 22,0 & 10,1 & 0,45 \\
\hline Ensemble & 20658 & 18,9 & 8,3 & 0,00 \\
\hline
\end{tabular}

Lecture: les scores obtenus aux tests de mémoire et de fonctions exécutives déclinent avec l'âge tandis qu'ils augmentent avec le niveau d'éducation.

Source : enquête Share, 2004.

distingue une première catégorie où l'on trouve l'âge et l'éducation et une deuxième catégorie contenant des variables de contrôle, parmi lesquelles des variables binaires par pays, le genre, l'origine et des maladies et accidents cérébraux. D'autre part, on distingue des variables explicatives supposées affecter la performance cognitive individuelle dont : les années écoulées depuis la fin de la vie active, l'exercice d'une ou de plusieurs activités non professionnelles ou d'une activité physique et deux autres facteurs indiquant si le répondant vit seul ou s'il souffre d'une mobilité réduite.

L'âge et l'éducation sont supposés être les facteurs déterminants des capacités cognitives. Exprimés touts les deux en forme logarithmique et en déviation par rapport à leur moyenne, ils sont au départ des variables continues définies en termes d'années. Dans la première vague de Share, chaque individu est interrogé sur le dernier diplôme obtenu à l'issue de ses études. C'est donc à partir de ces informations, détaillées et spécifiques pour chaque pays, qu'une variable «années d'éducation» a été calculée par l'équipe de Share, en suivant la Classification Internationale Type de l'Éducation (ISCED-97) (OCDE, 1999). Cette variable doit dès lors être considérée comme représentative des années théoriques d'éducation et pas comme des années effectives (5).
De plus, des variables dichotomiques (binaires) ont été intégrées dans le modèle à titre de variables de contrôle. Elles incluent tout d'abord des variables binaires par pays qui doivent capturer les différences résultant de particularités culturelles ou linguistiques. Les réponses aux deux tests cognitifs considérés ici sont en effet sensibles à ces facteurs, de même que le fait d'avoir comme langue maternelle la langue utilisée lors de l'entrevue, qui sera prise en compte indirectement par une variable indiquant si la personne interrogée est d'origine étrangère. Le genre est également introduit, afin de saisir l'effet potentiel des parcours de vie différents entre hommes et femmes. Une variable supplémentaire indique si l'individu souffre de maladies chroniques (au moins deux, sur une liste de quinze incluant l'hypertension, le cholestérol, le diabète, l'asthme, l'ostéoporose, l'arthrite, etc.).

Nous avons également inclus deux autres variables qui prennent plusieurs modalités: d'une part, l'attitude de la personne interrogée face à l'enquêteur tel que rapportée par l'enquêteur luimême sur la base de sa perception de la volonté à répondre de l'enquêté et d'autre part, une série de variables qui permettent de situer l'individu et le ménage dont il fait partie dans l'un des quartiles correspondant à la distribution de la richesse nette dans le pays correspondant. Cette variable est construite à partir des informations sur les actifs patrimoniaux des ménages disponibles également dans l'enquête Share.

De plus, trois variables binaires représentent des problèmes de santé en prise directe avec le fonctionnement cognitif : avoir ou non subi une attaque cérébrale, avoir ou non été victime d'une tumeur au cerveau et souffrir ou non de la maladie de Parkinson.

Trois autres variables sont également reprises dans cette catégorie en tant qu'indicateurs de facteurs liés à l'état de santé mentale et pouvant avoir une influence directe sur les performances lors de tests cognitifs : la prise régulière d'antidépresseurs au moment de l'enquête, le fait d'avoir séjourné par le passé dans un institut psychiatrique et la détection d'un certain nombre de symptômes relatifs à l'état de dépression.

Cette dernière variable a été construite dans le cadre de Share sur la base de l'échelle de dépression Euro-D, basée elle-même sur les répon-

5. Pour plus de détails sur la construction de cette variable cf. : www.share-project.org. 
ses données par les enquêtés à une batterie de questions permettant la détection d'un certain nombre de manifestations telles que le pessimisme, les tendances suicidaires, les sentiments de culpabilité, l'insomnie, le manque d'intérêt, l'irritabilité... Euro-D est une variable binaire dont la valeur unitaire correspond aux cas où le nombre de ces manifestations est supérieur à quatre, ce nombre étant considéré comme le seuil probable de l'état de dépression (6)

Comme indiqué précédemment, la réserve cognitive est potentiellement affectée par d'autres facteurs, caractéristiques individuelles associées au style de vie, en particulier par l'exercice d'activités, professionnelles ou non. L'enquête Share nous offre la possibilité de prendre en compte toute une série de facteurs explicatifs potentiels. Notre choix s'est porté sur trois types d'indicateurs.

En premier lieu, une série de variables dichotomiques indiquent si le répondant est toujours actif ou s'il l'a été, et dans ce cas s'il a arrêté son activité professionnelle au cours des cinq dernières années ou avant, par tranches de cinq ans. Ces variables sont autant de modalités d'une même variable censée synthétiser les différentes situations en relation avec la vie active et le départ à la retraite. Elles devraient nous permettre d'identifier notamment l'effet de la retraite anticipée sur les fonctions cognitives, dans la mesure où des personnes du même âge ayant quitté ou pas la vie active font partie de l'échantillon de Share.

En deuxième lieu, nous avons choisi une variable binaire décrivant le fait d'exercer ou d'avoir exercé au cours du dernier mois une activité ou une occupation non professionnelle. Ce type d'activité inclut le bénévolat, les activités caritatives, l'aide à une personne âgée ou handicapée, l'aide à un membre de la famille ou à un voisin, la participation à une formation ou à des cours, l'appartenance à un club sportif ou social et la participation à une organisation religieuse ou politique.

De manière similaire, la pratique d'activités demandant un effort physique modéré (le jardinage, le nettoyage de la voiture ou la promenade) ou vigoureux (un sport) est représentée à l'aide d'une série de variables binaires indiquant la fréquence ( « rarement ou pas du tout», « d'une à trois fois par mois », « une fois par semaine » et «plus d'une fois par semaine ») à laquelle la personne interrogée accomplit ces exercices.
Enfin, deux autres variables binaires sont prises en compte dans le modèle pour tester, d'une part, l'effet potentiel d'une mobilité réduite sur le fonctionnement cognitif et, d'autre part, celui dû à l'isolement social. La première a été construite à partir des réponses données par les personnes interrogées à une série de questions s'intéressant aux difficultés éventuelles rencontrées dans la vie quotidienne, telles que marcher 100 mètres, rester assis pendant deux heures, monter plusieurs volées d'escalier sans se reposer... (la liste complète contient dix items). La variable correspondante, mobilité réduite, prend la valeur unitaire quand la personne interrogée répond affirmativement à une ou plus de ces questions. Quant à la variable représentative de l'isolement social, elle est basée uniquement sur le fait que la personne vit seule ou non. Bien entendu, cette variable ne donne pas une certitude sur la situation d'isolement social effectif de la personne enquêté, mais uniquement un signe apparent.

\section{Biais potentiels dans l'analyse empirique}

Avant de procéder à la présentation des résultats de cette étude il est nécessaire d'évoquer ici une difficulté méthodologique qui pourrait en limiter la portée.

Share est un projet longitudinal et les répondants seront réinterrogés tous les deux ans pour les vagues suivantes (la deuxième vague ayant lieu en 2006-2007). Lorsque les données des vagues successives seront disponibles, il sera possible d'analyser les trajectoires individuelles en prêtant attention à la séquence des événements : états de santé, physique et mentale, situation sur le marché de travail, évolution des capacités cognitives, participation aux activités non professionnelles, entourage familial et événements de la vie. Le simple énoncé de ces aspects, qui font référence aux dimensions de la vie de chaque individu, soulève une difficulté de type méthodologique : la prise en compte des différents liens potentiels de causalité entre ces différentes dimensions.

Étant donné les informations dont nous disposons, issues de la première vague de Share, notre démarche est ici fondée sur un lien de causalité directionnel, les fonctions cognitives étant considérées comme variables endogènes dans le modèle. D'autres liens potentiels pourraient être

6. Pour plus de détails sur la construction de la variable Euro-D, cf. Prince et al. (1999a, 1999b). 
étudiés à partir de données longitudinales. Ces liens mettraient l'accent sur le rôle de la réserve cognitive sur l'exercice d'activités et des occupations diverses, mais également sur l'apparition et la détection de maladies neurologiques comme la maladie d'Alzheimer.

Plus concrètement, la disponibilité de données issues des vagues successives de Share permettra de réaliser une étude longitudinale et de s'attaquer à deux types de biais potentiels dans l'étude des performances cognitives : l'endogénéité et le biais dû à une variable omise dans le modèle.

D’une part, l'endogénéité potentielle serait due au lien de causalité entre le mauvais fonctionnement cognitif et la décision du départ à la retraite : les individus possédant une meilleure performance cognitive travaillent-ils plus longtemps ou, au contraire, le départ à la retraite accélère-t-il le vieillissement cognitif ? Ces deux phénomènes agissent sans doute de concert.

D'autre part, un biais de variable omise serait dû au fait que les départs anticipés à la retraite pourraient être fortement corrélés avec le type d'occupation exercé pendant la vie active. Le modèle estimé ici ne tient pas compte de l'effet du type de profession exercée sur les performances cognitives et cette omission est susceptible d'engendrer une corrélation fallacieuse entre ces dernières et la retraite. Il est en effet plus que probable que le type de profession influence la décision de quitter la vie active et joue également un rôle non négligeable sur les fonctions cognitives. Par exemple, si une profession manuelle est corrélée positivement au départ à la retraite et négativement aux performances cognitives, l'omission de la variable «profession manuelle» dans le modèle engendrerait une surestimation de l'effet de la retraite. De ce point de vue également, la dimension longitudinale de Share devrait permettre de tenir compte de ce biais potentiel et de le corriger.

\section{Des effets marqués mais attendus des différentes variables hormis pour le fait d'être atteint de maladie chronique}

À partir de l'échantillon initial de Share 2004 dans dix pays européens, nous avons retiré un certain nombre d'observations pour lesquelles des variables clefs du modèle (tests cognitifs, éducation et âge) étaient manquantes ou contenaient des erreurs. Ainsi, 20658 observations individuelles, personnes âgées de 50 ans ou plus, ont été retenues à partir des 22777 observations disponibles au départ (7)
Les coefficients associés aux variables âge et années d'éducation ont les signes attendus pour les trois modèles (respectivement négatif et positif), en plus d'être statistiquement significatifs dans la majorité des cas (cf. tableau 2). L'effet négatif de l'âge, aussi bien pour les termes de premier et de deuxième ordre, confirme le rôle du vieillissement sur le fonctionnement cognitif, effet qui tendrait à s'accélérer en vieillissant. Il en est de même en ce qui concerne l'effet positif des années d'éducation dans les deux cas, fonctions exécutives et mémoire épisodique, et pour les deux termes, de premier et deuxième ordre. Pour ce qui est du terme croisé, âge et années d'éducation, l'effet n'est pas significatif dans le cas des fonctions exécutives, mais positif et significatif en ce qui concerne la mémoire épisodique et pour l'indice global : le vieillissement cognitif est d'autant moins marqué que le niveau d'éducation est élevé.

On remarque immédiatement la courbure de l'hyperplan, négative en fonction de l'âge et positive suivant les années d'éducation. Les isoquants dessinent des courbes de niveau pour différents scores cognitifs globaux. La tangente de ces isoquants à chaque point permet de mesurer l'importance de l'éducation face au vieillissement cognitif. Ainsi, chaque année supplémentaire d'éducation servirait à « compenser » quatre années de vieillissement cognitif pour une personne âgée de 60 ans ayant eu dix années d'éducation (cf. graphique II).

À quelques exceptions près, maladie chronique notamment, l'ensemble des variables introduites dans les régressions se sont avérées avoir un effet significatif (y compris les variables binaires par pays, non reproduites ici) (cf. tableau 2). On remarque une différence significative entre femmes et hommes au niveau du test de mémoire épisodique et de l'indice global, ainsi qu'un effet négatif et significatif du fait d'être né à l'étranger pour chacun des modèles. De plus, les deux autres variables, «volonté de répondre à l'enquête » et « appartenance à un quartile déterminé dans la distribution du patrimoine net (dans le pays concerné) », ont les signes attendus. Enfin, au regard de l'indice global, la maladie de Parkinson, le fait d'avoir été victime d'une attaque cérébrale et les symptômes de dépression (Euro-D) jouent un rôle sur la réserve cognitive. La prise d'antidépresseurs

7. En ce qui concerne les informations manquantes pour les autres variables utilisées dans le modèle, variables de contrôle ou explicatives des distances à la frontière, elles ont donné lieu le cas échéant à une modalité spécifique reprise par une variable binaire supplémentaire. 
ou le fait d'avoir eu un séjour en institution psychiatrique ont également les effets attendus mais ces derniers ne sont pas significatifs. Ces différentes variables permettent d'ajuster l'emplacement de la frontière d'efficacité afin de procéder à la mesure des performances cognitives individuelles.

Les paramètres correspondant aux facteurs explicatifs affichent un signe négatif lorsque le facteur correspondant réduit la distance par rapport à la frontière, en d'autres termes il améliore les performances cognitives, tandis qu'un signe positif indique une détérioration de celles-ci (cf. tableau 3).

Le fait d'être inactif/inactive, de ne plus exercer une activité professionnelle, représenté par le temps écoulé depuis le départ à la retraite, est significativement lié à la réserve cognitive (positif sur la distance à la frontière), quelle que soit la mesure employée, de même que pour les personnes n'ayant jamais exercé d'activité professionnelle.

En outre, la pratique d'une activité non professionnelle, notamment le bénévolat sous toutes ces formes ainsi que l'aide aux proches ou le fait de suivre une formation, est significativement associée au maintien de la réserve cognitive.

Cette relation positive est également observée pour l'exercice d'activités physiques, modérées et/ou vigoureuses, et amplifiée en fonction de la fréquence de leur pratique. Cet effet dû à l'importance des activités physiques est d'ailleurs confirmé par l'effet associé à la variable mobilité réduite dans le cas des fonctions exécutives,

Tableau 2

Paramètres de la frontière stochastique

\begin{tabular}{|c|c|c|c|c|c|c|}
\hline \multirow{2}{*}{ Variables et coefficients } & \multicolumn{2}{|c|}{ Fonctions exécutives } & \multicolumn{2}{|c|}{ Mémoire épisodique } & \multicolumn{2}{|c|}{ Indice global } \\
\hline & \multicolumn{2}{|c|}{ Coefficient et $t$-ratio } & \multicolumn{2}{|c|}{ Coefficient et $t$-ratio } & \multicolumn{2}{|c|}{ Coefficient et $t$-ratio } \\
\hline $\begin{array}{l}\text { Âge et années d'éducation } \\
\text { Constante } \\
\text { Âge }\left(\ln x_{1}\right) \\
\text { Années d'éducation }\left(\ln x_{2}\right) \\
\left(\ln x_{1}\right)^{2} \\
\left(\ln x_{2}\right)^{2} \\
\left(\ln x_{1}\right)\left(\ln x_{2}\right)\end{array}$ & $\begin{array}{r}0,314^{*} \\
-0,343^{*} \\
0,197^{*} \\
-0,902^{*} \\
0,056^{*} \\
-0,010\end{array}$ & $\begin{array}{r}(33,7) \\
(-20,3) \\
(30,0) \\
(-10,1) \\
(15,9) \\
(-0,4)\end{array}$ & $\begin{array}{r}0,374^{*} \\
-0,467^{*} \\
0,186^{*} \\
-0,970^{*} \\
0,051^{*} \\
0,120^{*}\end{array}$ & $\begin{array}{r}(36,6) \\
(-27,3) \\
(27,8) \\
(-10,2) \\
(13,9) \\
(4,8)\end{array}$ & $\begin{array}{r}0,737^{\star} \\
-0,993^{\star} \\
0,477^{\star} \\
-2,263^{\star} \\
0,133^{*} \\
0,142^{\star}\end{array}$ & $\begin{array}{r}(33,7) \\
(-29,2) \\
(36,6) \\
(-12,1) \\
(18,9) \\
(3,0)\end{array}$ \\
\hline \multicolumn{3}{|l|}{$\begin{array}{l}\text { Variables de contrôle (binaires) } \\
\text { Binaires par pays }\end{array}$} & \multicolumn{2}{|c|}{ Oui } & \multicolumn{2}{|c|}{ Oui } \\
\hline $\begin{array}{l}\text { Genre féminin } \\
\text { Origine étrangère } \\
\text { Maladie chronique }\end{array}$ & $\begin{array}{c}0,002 \\
-0,097^{*} \\
0,004\end{array}$ & $\begin{array}{r}(0,5) \\
(-12,6) \\
(0,9)\end{array}$ & $\begin{array}{c}0,083^{*} \\
-0,046^{*} \\
0,005\end{array}$ & $\begin{array}{r}(19,3) \\
(-6,0) \\
(1,0)\end{array}$ & $\begin{array}{c}0,106^{\star} \\
-0,175^{\star} \\
0,015\end{array}$ & $\begin{array}{r}(12,6) \\
(-11,5) \\
(1,7)\end{array}$ \\
\hline \multicolumn{7}{|l|}{ Volonté de répondre à l'enquête } \\
\hline $\begin{array}{l}\text { Très grande } \\
\text { Grande } \\
\text { Moyenne } \\
\text { Faible }\end{array}$ & $\begin{array}{l}-0,049^{\star} \\
-0,099^{\star} \\
-0,187^{\star}\end{array}$ & $\begin{array}{r}(-10,4) \\
(-14,1) \\
(-8,5)\end{array}$ & $\begin{array}{l}-0,045^{*} \\
-0,100^{*} \\
-0,120^{*}\end{array}$ & $\begin{array}{r}c e \\
(-9,4) \\
(-13,9) \\
(-5,6)\end{array}$ & $\begin{array}{r}R \epsilon \\
-0,113^{\star} \\
-0,231^{*} \\
-0,332^{\star}\end{array}$ & $\begin{array}{r}(-12,3) \\
(-16,3) \\
(-7,7)\end{array}$ \\
\hline \multicolumn{7}{|l|}{ Patrimoine (quartiles) } \\
\hline $\begin{array}{l}2^{e} \\
3^{e} \\
4^{e}\end{array}$ & $\begin{array}{l}0,028^{*} \\
0,043^{*} \\
0,054^{\star}\end{array}$ & $\begin{array}{l}(4,9) \\
(7,3) \\
(9,0)\end{array}$ & $\begin{array}{l}0,012 \\
0,024^{\star} \\
0,021^{*}\end{array}$ & $\begin{array}{l}(2,0) \\
(4,1) \\
(3,5)\end{array}$ & $\begin{array}{l}0,047^{*} \\
0,084^{\star} \\
0,091^{\star}\end{array}$ & $\begin{array}{l}(4,1) \\
(7,2) \\
(7,6)\end{array}$ \\
\hline \multicolumn{7}{|l|}{ Maladies et accidents cérébraux } \\
\hline $\begin{array}{l}\text { Attaque cérébrale } \\
\text { Maladie de Parkinson } \\
\text { Tumeur au cerveau }\end{array}$ & $\begin{array}{l}-0,054^{*} \\
-0,073 \\
-0,100\end{array}$ & $\begin{array}{l}(-4,9) \\
(-2,5) \\
(-1,6)\end{array}$ & $\begin{array}{r}-0,041^{*} \\
-0,049 \\
0,005\end{array}$ & $\begin{array}{r}(-3,5) \\
(-1,6) \\
(0,1)\end{array}$ & $\begin{array}{l}-0,124^{\star} \\
-0,183^{\star} \\
-0,135\end{array}$ & $\begin{array}{l}(-5,3) \\
(-3,1) \\
(-1,1)\end{array}$ \\
\hline \multicolumn{7}{|l|}{ Santé mentale } \\
\hline $\begin{array}{l}\text { Prise d'anti- dépresseurs } \\
\text { Séjour en institut psychiatrique }\end{array}$ & $\begin{array}{l}-0,023 \\
-0,011\end{array}$ & $\begin{array}{l}(-2,2) \\
(-0,8)\end{array}$ & $\begin{array}{l}-0,015 \\
-0,029\end{array}$ & $\begin{array}{l}(-1,5) \\
(-1,9)\end{array}$ & $\begin{array}{l}-0,048 \\
-0,037\end{array}$ & $\begin{array}{l}(-2,4) \\
(-1,3)\end{array}$ \\
\hline \multicolumn{7}{|l|}{ Symptômes dépressifs } \\
\hline $\begin{array}{l}\text { Euro- } D=0 \\
\text { Euro- } D=1\end{array}$ & $\begin{array}{r}R \\
-0,020^{*}\end{array}$ & $e_{(-3,7)}$ & $\begin{array}{r}R \\
-0,040^{*}\end{array}$ & $c e$ & $\begin{array}{r}R \epsilon \\
-0,070^{*}\end{array}$ & $(-6,7)$ \\
\hline
\end{tabular}

Lecture : les paramètres rapportés aux tableaux 2 et 3 et correspondant à chacun des modèles ont été estimés simultanément utilisant le programme Frontier (Coelli, 1994). Ils sont affichés séparément pour des raisons de présentation.

* Significatif au seuil de $1 \%$.

Champ : individus âgés de 50 ans et plus dans 10 pays européens en 2004.

Source : enquête Share, 2004. 


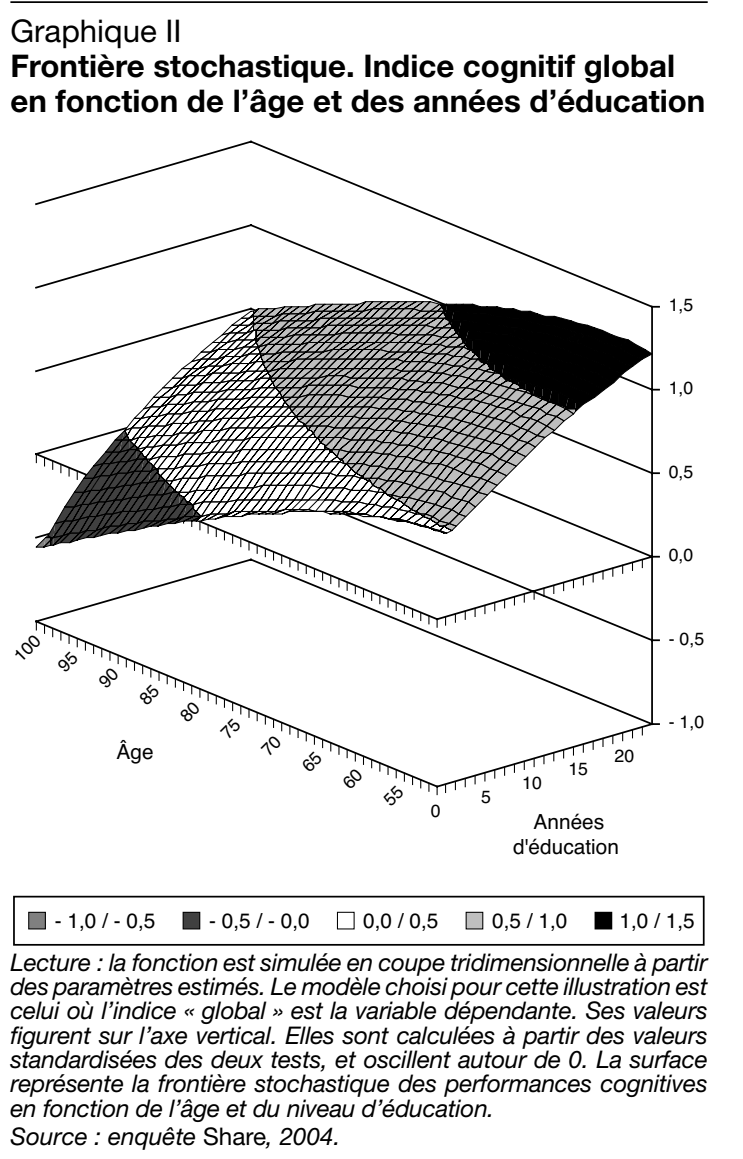

la mémoire épisodique répondant au contraire de manière favorable (par un signe négatif). Ce résultat est une confirmation du fait que chaque test appelle à des compétences cognitives différentes. Finalement, le fait de vivre dans un ménage isolé a pour conséquence une diminution significative des performances cognitives quel que soit le score analysé.

Afin d'illustrer l'importance des relations entre ces facteurs et la réserve cognitive, une simulation est réalisée sur la base des individus âgés de 60 ans dans l'échantillon de Share. Ces résultats sont présentés en terme de vieillissement cognitif, c'est-à-dire l'équivalent en années de vieillissement gagnées ou perdues suite à une modification de l'une ou l'autre des variables explicatives (cf. tableau 4).

On procède en deux étapes. La première consiste à calculer, pour chaque individu âgé de 60 ans et pour chaque facteur, l'effet d'un changement de ce facteur, ceteris paribus, sur la réserve cognitive en utilisant les coefficients du modèle (cf. tableau 3). La seconde étape consiste à cal-

Tableau 3

\section{Facteurs explicatifs des performances cognitives}

\begin{tabular}{|c|c|c|c|c|c|c|}
\hline \multirow{3}{*}{\begin{tabular}{|l|} 
\\
Variables explicatives \\
Constante
\end{tabular}} & \multirow{2}{*}{\multicolumn{2}{|c|}{$\begin{array}{l}\text { Fonctions exécutives } \\
\text { Coefficient et } t \text {-ratio }\end{array}$}} & \multirow{2}{*}{\multicolumn{2}{|c|}{$\begin{array}{l}\text { Mémoire épisodique } \\
\text { Coefficient et } t \text {-ratio }\end{array}$}} & \multicolumn{2}{|c|}{ Indice global } \\
\hline & & & & & \multicolumn{2}{|c|}{ Coefficientet $t$-ratio } \\
\hline & $-3,384^{\star}$ & $(-8,6)$ & $-1,315^{\star}$ & $(-6,8)$ & $-1,185^{\star}$ & $(-4,5)$ \\
\hline \multicolumn{7}{|l|}{ Activité professionnelle } \\
\hline Actif & \multicolumn{2}{|c|}{ Référence } & \multicolumn{2}{|c|}{ Référence } & \multicolumn{2}{|c|}{ Référence } \\
\hline Inactif depuis 0 à 4 ans & $0,546^{\star}$ & $(9,7)$ & $0,299^{*}$ & $(7,4)$ & $0,425^{\star}$ & $(5,7)$ \\
\hline Inactif depuis 5 à 9 ans & $0,700^{*}$ & $(8,3)$ & $0,451^{*}$ & $(8,8)$ & $0,529^{*}$ & $(7,1)$ \\
\hline Inactif depuis 10 à 14 ans & $0,760^{\star}$ & $(11,8)$ & $0,508^{\star}$ & $(10,4)$ & $0,632^{*}$ & $(8,4)$ \\
\hline Inactif depuis 15 ans ou plus & $0,976^{\star}$ & $(10,6)$ & $0,677^{\star}$ & $(12,3)$ & $0,843^{\star}$ & $(10,0)$ \\
\hline N’a pas eu d'activité & $1,809^{*}$ & $(9,0)$ & $0,786^{\star}$ & $(12,9)$ & $1,203^{*}$ & $(10,4)$ \\
\hline Situation non rapportée & $1,640^{*}$ & $(10,2)$ & $1,090^{\star}$ & $(12,6)$ & $1,452^{*}$ & $(10,1)$ \\
\hline \multicolumn{7}{|l|}{ Activité non professionnelle } \\
\hline Aucune & \multicolumn{2}{|c|}{ Référence } & \multicolumn{2}{|c|}{ Référence } & \multicolumn{2}{|c|}{ Référence } \\
\hline Au moins une activité non-professionnelle & $-1,910^{*}$ & $(-9,4)$ & $-0,372^{\star}$ & $(-10,4)$ & $-0,815^{\star}$ & $(-10,0)$ \\
\hline \multicolumn{7}{|l|}{ Activités physiques... } \\
\hline ... vigoureuses : jamais ou presque & \multicolumn{2}{|c|}{ Référence } & \multicolumn{2}{|c|}{ Référence } & \multicolumn{2}{|c|}{ Référence } \\
\hline$\ldots$ vigoureuses : une à trois fois par mois & $-0,926^{\star}$ & $(-7,4)$ & $-0,332^{*}$ & $(-6,3)$ & $-0,579^{\star}$ & $(-7,0)$ \\
\hline$\ldots$ vigoureuses : une fois par semaine & $-0,788^{\star}$ & $(-8,8)$ & $-0,396^{\star}$ & $(-7,8)$ & $-0,635^{\star}$ & $(-7,5)$ \\
\hline ... vigoureuses : plus d'une fois par semaine & $-1,497^{\star}$ & $(-7,9)$ & $-0,318^{\star}$ & $(-9,4)$ & $-0,694^{*}$ & $(-8,4)$ \\
\hline ... modérées : jamais ou presque & \multicolumn{2}{|c|}{ Référence } & \multicolumn{2}{|c|}{ Référence } & \multicolumn{2}{|c|}{ Référence } \\
\hline ... modérées : une à trois fois par mois & $-1,121^{*}$ & $(-5,7)$ & $-0,322^{*}$ & $(-5,4)$ & $-0,619^{\star}$ & $(-6,8)$ \\
\hline ... modérées : une fois par semaine & $-0,948^{\star}$ & $(-7,1)$ & $-0,289^{\star}$ & $(-7,3)$ & $-0,512^{*}$ & $(-7,3)$ \\
\hline ... modérées : plus d'une fois par semaine & $-1,996^{\star}$ & $(-8,3)$ & $-0,354^{\star}$ & $(-9,0)$ & $-0,859^{\star}$ & $(-9,7)$ \\
\hline \multicolumn{7}{|l|}{ Autres facteurs } \\
\hline Mobilité réduite & $-0,255^{\star}$ & $(-6,6)$ & $0,142^{*}$ & $(6,5)$ & $0,132^{*}$ & $(3,8)$ \\
\hline Ménage isolé & $0,326^{\star}$ & $(5,8)$ & $0,219^{\star}$ & $(7,5)$ & $0,343^{\star}$ & $(7,5)$ \\
\hline \multicolumn{7}{|l|}{ Autres paramètres } \\
\hline$\sigma_{\varepsilon}^{2}=\sigma_{v}^{2}+\sigma_{u}^{2}$ & $1,263^{*}$ & $(9,5)$ & $0,726^{*}$ & $(12,0)$ & $1,779^{*}$ & $(11,5)$ \\
\hline$\gamma=\sigma_{u}^{2} / \sigma_{\varepsilon}^{2}$ & $0,958^{*}$ & $(204,2)$ & $0,955^{\star}$ & $(279,8)$ & $0,911^{*}$ & $(134,7)$ \\
\hline
\end{tabular}

Lecture : les paramètres rapportés aux tableaux 2 et 3 et correspondant à chacun des modèles ont été estimés simultanément utilisant le programme Frontier (Coelli, 1994). Ils sont affichés séparément pour des raisons de présentation.

* Significatif au seuil de $1 \%$.

Champ : individus âgés de 50 ans et plus dans dix pays européens en 2004. Source : enquête Share, 2004. 
Tableau 4

Effet des facteurs explicatifs, en années de vieillissement cognitif

\begin{tabular}{|c|c|c|c|}
\hline Facteurs explicatifs & Fonctions exécutives & Mémoire épisodique & Indice global \\
\hline $\begin{array}{l}\text { Activité professionnelle } \\
\text { Actif } \\
\text { Inactif depuis } 0 \text { à } 4 \text { ans } \\
\text { Inactif depuis } 5 \text { à } 9 \text { ans } \\
\text { Inactif depuis } 10 \text { à } 14 \text { ans } \\
\text { Inactif depuis } 15 \text { ans ou plus } \\
\text { N'a pas eu d'activité }\end{array}$ & $\begin{array}{c}\text { Référence } \\
1,30 \\
1,64 \\
1,78 \\
2,25 \\
3,97\end{array}$ & $\begin{array}{c}\text { Référence } \\
1,22 \\
1,54 \\
1,66 \\
2,10 \\
3,73\end{array}$ & $\begin{array}{c}\text { Référence } \\
1,03 \\
1,30 \\
1,41 \\
1,78 \\
3,13\end{array}$ \\
\hline $\begin{array}{l}\text { Activité non professionnelle } \\
\text { Aucune } \\
\text { Au moins une activité non professionnelle }\end{array}$ & $\begin{array}{c}\text { Référence } \\
-4,08\end{array}$ & $\begin{array}{l}\text { Référence } \\
-3,83\end{array}$ & $\begin{array}{l}\text { Référence } \\
-3,23\end{array}$ \\
\hline $\begin{array}{l}\text { Activités physiques... } \\
\ldots \text { vigoureuses : jamais ou presque } \\
\ldots \text { vigoureuses : une à trois fois par mois } \\
\ldots \text { vigoureuses : une fois par semaine } \\
\ldots \text { vigoureuses : plus d'une fois par semaine } \\
\ldots \text { modérées : jamais ou presque } \\
\ldots \text { modérées : une à trois fois par mois } \\
\ldots \text { modérées : une fois par semaine } \\
\ldots \text { modérées : plus d'une fois par semaine }\end{array}$ & $\begin{array}{l}\text { Référence } \\
-2,20 \\
-1,91 \\
-3,30 \\
\text { Référence } \\
-2,74 \\
-2,38 \\
-4,41\end{array}$ & $\begin{array}{l}\text { Référence } \\
-2,06 \\
-1,78 \\
-3,10 \\
\text { Référence } \\
-2,56 \\
-2,22 \\
-4,13\end{array}$ & $\begin{array}{l}\text { Référence } \\
-1,73 \\
-1,50 \\
-2,61 \\
\text { Référence } \\
-2,13 \\
-1,84 \\
-3,46\end{array}$ \\
\hline $\begin{array}{l}\text { Autres facteurs } \\
\text { Mobilité réduite } \\
\text { Ménage isolé }\end{array}$ & $\begin{array}{r}-0,64 \\
0,82\end{array}$ & $\begin{array}{l}0,60 \\
0,76\end{array}$ & $\begin{array}{l}0,51 \\
0,65\end{array}$ \\
\hline
\end{tabular}

Lecture : un individu de 60 ans retarde son vieillissement cognitif de 1,3 année s'il est toujours actif professionnellement par rapport à un individu inactif depuis moins de 5 ans.

Champ : personnes de 60 ans.

Source : enquête Share, 2004

culer combien d'années de vieillissement cognitif « normal » donneraient le même résultat pour un individu âgé de 60 ans. À cet effet, chaque individu est projeté sur la frontière d'efficacité (cf. graphique II) en tenant compte de ses caractéristiques spécifiques, années d'éducation, sexe, etc., et de l'ensemble des paramètres.

On remarque que les résultats sont similaires pour les trois scores cognitifs. Le vieillissement cognitif chez une personne inactive depuis quelques années (moins de 5 ans) par rapport à un travailleur du même âge serait de 1,30 année si l'on se réfère aux fonctions exécutives, et de 1,22 année pour la mémoire épisodique (cf. tableau 4). Cet effet s'accroît quand la personne a quitté la vie professionnelle depuis plus longtemps ou n'a jamais exercé une activité professionnelle rémunérée.

De plus, la pratique d'activités non professionnelles ou d'activités physiques est associée de manière non négligeable au vieillissement cognitif: elle retarde celui-ci de 3,23 années dans le premier cas et de 1,84 dans le deuxième, pour une activité physique modérée. Ces résultats confirment les observations similaires mises en évidence par Menec (2003) qui montrent une relation entre l'exercice d'activités quotidiennes et le vieillissement cognitif.

Une vue d'ensemble de ces résultats indique l'existence d'une relation significative entre la retraite et les activités non professionnelles, et le fonctionnement cognitif des individus âgés de plus de 50 ans.

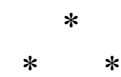

Néanmoins, ces résultats se doivent d'être nuancés étant donné les limitations et les biais potentiels du modèle. Il est fort probable en effet que les capacités cognitives affectent la décision de se retirer de la vie active et inversement. Cette étude constitue une première exploration de ces relations à partir des données de Share, elle devrait être suivie par d'autres afin de déterminer les liens de causalité subjacents et de distinguer chacun de ces effets. La mise à disposition des données des vagues successives de cette enquête, prévues tous les deux ans auprès des mêmes individus, pourrait permettre d'y répondre. 
Pour la réalisation de cette recherche, nous nous sommes servis des données issues de la première version (Release 1) de la vague 1 de l'enquête Share réalisée en 2004. Celle-ci est préliminaire et pourrait contenir des erreurs qui seront corrigées dans des versions ultérieures. La collecte des données de Share a été principalement financée par le $5^{e}$ Programme Cadre de la Communauté Européenne (Projet QLK6-CT-2001-00360 sur le thème de la qualité de la vie). Des fonds proviennent également du US National Institute on Aging (U01 AG09740-13S2, P01 AG005842, P01 AG08291, P30 AG12815, Y1-AG-4553-01 et OGHA 04-064). La collecte des données a été financée par des institutions nationales en Autriche (Austrian Science Foundation, FWF), en Belgique (Politique Scientifique Fédérale) et en Suisse (BBW/OFES/UFES). Pour la France, elle a bénéficié de financements complémentaires apportés par la Cnav, la Cnam, le Cor, la Drees, la Dares, la Caisse des Dépôts et Consignations et le Commissariat Général du Plan. Les données et la méthodologie de l'enquête sont respectivement présentées dans Börsch-Supan et al. (2005) et BörschSupan et Jürges (2005).

\section{BIBLIOGRAPHIE}

Adam S., Bay Ch., Bonsang E., Germain S. et Perelman S. (2006), « Occupational Activities and Cognitive Reserve : a Frontier Approach Applied to the Survey on Health, Ageing, and Retirement in Europe (Share) », CREPP, Université de Liège, DP 2006/05.

Aigner D.J., Lovell C.A.K. et Schmidt P. (1977), «Formulation and Estimation of Stochastic Frontier Production Function Models ", Journal of Econometrics, vol. 6, n ${ }^{\circ}$ 1, pp. 21-37.

Anderson N.D.et Craik F.I.M. (2000), « Memory in the Aging Brain », in The Oxford Handbook of Memory, Tulving E. et Craik F.I.M. éds., pp. 411425, Oxford : Oxford University Press.

Battese G.E. et Coelli T.J. (1995), «A Model for Technical Inefficiency Effects in a Stochastic Frontier Production Function for Panel Data », Empirical Economics, vol. 20, n 2, pp. 325-332.

Blöndal S.etScarpetta S. (1998), « The Retirement Decision in OECD Countries », OECD Economics Department Working Papers, $\mathrm{n}^{\circ} 202$.

Börsch-Supan A., Brugiavini A., Jürges H., Mackenbach J., Siegrist J. et Weber G. (éds.) (2005), Health, Ageing and Retirement in Europe. First Results from the Survey of Health, Ageing and Retirement in Europe, Mannheim, MEA. Téléchargeable sur : http :// www. SHARE-project.org/DocumentationChapters.html.

Börsch-Supan A. et Jürges H. (éds.) (2005), The Survey of Health, Ageing and Retirement in Europe - Methodology, Mannheim, MEA.
Charnes A. Cooper W.W. et Rhodes E.L. (1978), "Measuring the Efficiency of Decision Making Units » European Journal of Operational Research, vol. 2, n 6, pp. 429-444.

Coelli T.J. (1994), A Guide to FRONTIER Version 4.1: A Computer Program for Stochastic Production and Cost Function Estimation, Department of Econometrics, University of New England.

Coelli T.J., Rao D.S.P., O'Donnell C.J. et Battese G.E. (2005), An Introduction to Efficiency and Productivity Analysis, $2^{\mathrm{e}}$ édition, Springer, New York.

Dixon R.A., Bäckman L. et Nilsson L.G. (2004), New Frontiers in Cognitive Aging, Oxford University Press, Oxford.

Kumbhakar S.C. et Lovell C.A.K. (2000), Stochastic Frontier Analysis, Cambridge University Press.

Le Carret N., Lafont S., Letenneur L., Dartigues F., Mayo W. et Fabrigoule C. (2003), "The Effect of Education on Cognitive Performances and its Implication for the Constitution of the Cognitive Reserve », Developmental Neuropsychology, vol. 23, $\mathrm{n}^{\circ} 3$, pp. 317-337.

Meeusen W. et Van den Broeck J. (1977), «Efficiency Estimation from Cobb-Douglas Production Functions with Composed Error», International Economic Review, vol. 18, $\mathrm{n}^{\circ} 2$, pp. 435-444.

Menec V. (2003), «The Relation Between Everyday Activities and Successful Aging: A 
6-Year Longitudinal Study », The Journals of Gerontology. Psychological Sciences and Social Sciences, vol. 58B, n 2, pp. S74-S82.

Newson R.S. et Kemps E.B. (2005), « General Lifestyle Activities as a Predictor of Current Cognition and Cognitive Change in Older Adults : A Cross-Sectional and Longitudinal Examination "), The Journal of Gerontology. Psychological Sciences and Social Sciences, vol. 60B, n ${ }^{\circ} 3$, pp. 113-120.

OECD (1999), Classifying Educational Programmes. Manual for ISCED-97 Implementation in OECD Countries - 1999 Edition, Paris.

Prince M.J., Beekman A.T., Deeg, D. J., Fuhrer R., Kivela S.L., Lawlor B.A. et al. (1999a), «Depression Symptoms in Late Life Assessed Using the EURO-D Scale », The British Journal of Psychiatry, vol. 174, n 4, pp. 339-345.

Prince M.J., Reischies F., Beekman A. T.F., Fuhrer R., Jonker C., Kivela S.L. et al. (1999b), «Development of the EURO-D Scale : A European Union Initiative to Compare Symptoms of Depression in 14 European Centres », The British Journal of Psychiatry, vol. $174, \mathrm{n}^{\circ} 4$, pp. 330-338.

Prull M.W., Gabrieli J.D.E. et Bunge S. A. (2000), "Age-Related Changes in Memory: A Cognitive Neuroscience Perspective », in Handbook of Aging and Cognition II, Craik F.I.M. et Salthouse T.A. (éds.), pp. 91-153, Mahwah, NJ : Lawrence Erlbaum Associates.
Scarmeas N. et Stern Y. (2003), « Cognitive Reserve and Lifestyle », Journal of Clinical and Experimental Neuropsychology, vol. 25, $n^{\circ} 5$, pp. 625-633.

Schooler C., Mulatu M.S. et Oates G. (1999), "The Continuing Effects of Substantively Complex Work on the Intellectual Functioning of Older Workers ", Psychology and Aging, vol. 14, $n^{\circ}$ 3, pp. 483-506.

Souchay C., Isingrini M. et Espagnet L. (2000), « Aging, Episodic Memory, Feeling-of-Knowing, and Frontal Functioning ", Neuropsychology, vol. 14, no 2, pp. 299-309.

Stern Y. (2002), «What is Cognitive Reserve? Theory and Research Application of the Reserve Concept », Journal of the International Neuropsychological Society, vol. $8, n^{\circ} 3$, pp. 448-460.

Stern Y. (2003), «The Concept of Cognitive Reserve : A catalyst for Research », Journal of Clinical and Experimental Neuropsychology, vol. $25, n^{\circ} 5$, pp. 589-593.

Wilson R.S., Mendes de Leon C. F., Barnes L. L., Schneider J.A., Bienias J.L., Evans D. A. et Bennet D.A. (2002), "Participation in Cognitively Stimulating Activities and Risk of Incident Alzheimer Disease ", Journal of the American Medical Association, vol. 287, $\mathrm{n}^{\circ} 6$, pp. 742-748. 